ARTICLE

https://doi.org/10.1038/s41467-019-12530-w

\title{
Synthesis of magnesium-nitrogen salts of polynitrogen anions
}

Dominique Laniel (1) ${ }^{1 \star}$, Bjoern Winkler ${ }^{2}$, Egor Koemets ${ }^{3}$, Timofey Fedotenko ${ }^{1}$, Maxim Bykov (D) ${ }^{3}$, Elena Bykova (1) ${ }^{4}$, Leonid Dubrovinsky (1) ${ }^{3} \&$ Natalia Dubrovinskaia (i) ${ }^{1}$

The synthesis of polynitrogen compounds is of fundamental importance due to their potential as environmentally-friendly high energy density materials. Attesting to the intrinsic difficulties related to their formation, only three polynitrogen ions, bulk stabilized as salts, are known. Here, magnesium and molecular nitrogen are compressed to about $50 \mathrm{GPa}$ and laser-heated, producing two chemically simple salts of polynitrogen anions, $\mathrm{MgN}_{4}$ and $\mathrm{Mg}_{2} \mathrm{~N}_{4}$. Singlecrystal X-ray diffraction reveals infinite anionic polythiazyl-like 1D N-N chains in the crystal structure of $\mathrm{MgN}_{4}$ and cis-tetranitrogen $\mathrm{N}_{4}{ }^{4-}$ units in the two isosymmetric polymorphs of $\mathrm{Mg}_{2} \mathrm{~N}_{4}$. The cis-tetranitrogen units are found to be recoverable at atmospheric pressure. Our results respond to the quest for polynitrogen entities stable at ambient conditions, reveal the potential of employing high pressures in their synthesis and enrich the nitrogen chemistry through the discovery of other nitrogen species, which provides further possibilities to design improved polynitrogen arrangements.

\footnotetext{
${ }^{1}$ Material Physics and Technology at Extreme Conditions, Laboratory of Crystallography, University of Bayreuth, 95440 Bayreuth, Germany. ${ }^{2}$ Institut für Geowissenschaften, Abteilung Kristallographie, Johann Wolfgang Goethe-Universität Frankfurt, Altenhöferallee 1, D-60438 Frankfurt am Main, Germany. ${ }^{3}$ Bayerisches Geoinstitut, University of Bayreuth, 95440 Bayreuth, Germany. ${ }^{4}$ Photon Science, Deutsches Elektronen-Synchrotron, Notkestrasse 85,22607 Hamburg, Germany. *email: dominique.laniel@uni-bayreuth.de
} 
olynitrogen entities are recognized to be ideal constituents of high energy density materials (HEDM) on account of the tremendous energy released upon the conversion of singly or doubly bonded $\mathrm{N}-\mathrm{N}$ atoms to triple-bonded molecular nitrogen ${ }^{1}$. As a direct consequence of their energetic potential, these low order bonds are highly unstable. So far, only three homonuclear polynitrogen species have been bulk stabilized at ambient conditions: $\mathrm{N}_{3}{ }^{-}, \mathrm{N}_{5}{ }^{+}$, and $\mathrm{N}_{5}{ }^{-2}$. In particular, the stabilization and isolation of the $\mathrm{N}_{5}{ }^{+}$and $\mathrm{N}_{5}{ }^{-}$ions is arduous and typically achieved in salts with a complex, multi-elements, counter ion ${ }^{3-8}$. Other polynitrogen units are known to exist, such as $\mathrm{N}_{4}^{+}$and $\mathrm{N}_{4}$, but they are deemed unstable as they have a lifetime below the millisecond threshold ${ }^{9}$ and can only be kept intact by being trapped in inert matrices at ultralow temperatures ${ }^{10,11}$.

The application of pressure opens up unique possibilities to explore and produce polynitrogen entities. Indeed, pressure generally promotes electronic delocalization and thus favors the formation of extended and polymeric networks. As a matter of fact, the only bulk stable neutral energetic polynitrogen specie, cubic-gauche polymeric nitrogen $(\mathrm{cg}-\mathrm{N})$, was synthesized under pressure, at $110 \mathrm{GPa}^{12}$. In that same pressure regime, other polynitrogen anions were discovered, such as infinite $1 \mathrm{D}$ armchair chains (in $\mathrm{FeN}_{4}$ and $\left.\operatorname{ReN}_{8} \cdot x \mathrm{~N}_{2}\right)^{13,14}$ and the pentazolate ring $\left(\mathrm{N}_{5}{ }^{-}\right)$, found in $\mathrm{CsN}_{5}{ }^{15}$. Despite numerous attempts, none of these compounds could be recovered at ambient conditions. The sole exception is the $(\mathrm{Li})^{+}\left(\mathrm{N}_{5}\right)^{-}$salt, produced near $50 \mathrm{GPa}$ and retrieved at ambient conditions ${ }^{16}$. Unlike the vast majority of poly- $\mathrm{N}$ ions formed through classical chemistry methods, their pressure-formed counterparts have the significant advantage of being extremely simple, typically with a single element acting as the counter ion.

Atomistic model calculations highlight the outstanding potential of high pressure experiments, as a plethora of polynitrogen entities have been predicted to be stable ${ }^{17-19}$. Among them, the compression of magnesium and nitrogen was predicted to produce the $\mathrm{MgN}_{3}, \mathrm{MgN}_{4}$, and $\mathrm{MgN}_{10}$ salts comprised of exotic anionic benzene-like $\mathrm{N}_{6}$ rings, infinite $1 \mathrm{D}$ armchair chains and pentazolates, respectively ${ }^{17-19}$. To the best of our knowledge, these predictions have not been tested until now.

Here, we demonstrate that the $(\mathrm{Mg})^{2+}\left(\mathrm{N}_{4}\right)^{2-}$ and the $\left(\mathrm{Mg}_{2}\right)^{4+}\left(\mathrm{N}_{4}\right)^{4-}$ salts are synthesized by compressing and laserheating magnesium and molecular nitrogen samples above
$50 \mathrm{GPa}$. Single-crystal X-ray diffraction measurements establish polynitrogen entities, namely infinite anionic polythiazyl-like 1D $\mathrm{N}-\mathrm{N}$ chains and cis-tetranitrogen $\mathrm{N}_{4}{ }^{4-}$ species, to compose the $\mathrm{MgN}_{4}$ and $\mathrm{Mg}_{2} \mathrm{~N}_{4}$ solids, respectively. Upon the full pressure release, the $\beta-\mathrm{Mg}_{2} \mathrm{~N}_{4}$ compound undergoes an isosymmetric phase transition into the $\alpha-\mathrm{Mg}_{2} \mathrm{~N}_{4}$ salt, also comprised of cistetranitrogen $\mathrm{N}_{4}{ }^{4-}$ units. These results demonstrate the recoverability to ambient conditions of the pressure-produced $\mathrm{N}_{4}{ }^{4-}$ entity, emphasizing the potential and importance of the high pressure approach for the discovery and synthesis of improved polynitrogen species.

\section{Results and discussion}

Synthesis and characterization of the Mg-N compounds. With the goal of synthesizing nitrogen-rich $\mathrm{Mg}-\mathrm{N}$ solids, we compressed micrometer-size pure magnesium pieces surrounded by a large volume of molecular nitrogen-used as both a pressure transmitting medium and a reagent-up to about $60 \mathrm{GPa}$ in four diamond anvil cells (DACs). To facilitate a chemical reaction, samples were laser-heated using YAG lasers at pressures of 28.0, $33.0,43.4,52.2,52.4,52.7$, and $58.1 \mathrm{GPa}$. The specific pressuretemperature paths followed for all samples are summarized in Supplementary Table 1. Metallic Mg served as a YAG laser absorber. Laser-heating at pressures below 52.2 GPa resulted in the formation of a previously known compound, $\mathrm{Mg}_{3} \mathrm{~N}_{2}$ (space group $\mathrm{C} 2 / \mathrm{m}),{ }^{20}$ identified by both X-ray diffraction and Raman spectroscopy measurements (see Supplementary Figures 1 and 2 as well as Supplementary Table 2). Above the threshold pressure of $52.2 \mathrm{GPa}$, heating the samples to at least $1850 \mathrm{~K}$ led to the growth of the $\mathrm{Mg}$ piece-supposedly due to nitrogen diffusing into it-, the appearance of two sets of Raman modes and diffraction lines which did not correspond to known phases of either $\mathrm{Mg}_{3} \mathrm{~N}_{2}{ }^{20}$, pure $\mathrm{Mg}^{21}$, or pure $\mathrm{N}_{2}{ }^{22,23}$ (Supplementary Figs. 4-7). Single-crystal X-ray diffraction revealed the crystal structures of two compounds with compositions $\mathrm{Mg}_{2} \mathrm{~N}_{4}$ and $\mathrm{MgN}_{4}$ (crystallographic data are given in Supplementary Table 3 and Supplementary Table 4).

The $\mathrm{MgN}_{4}$ compound has an orthorhombic structure (Ibam space group) with the lattice parameters $a=3.5860$ (13) $\AA, b=$ $7.526(3) \AA$ and $c=5.1098(17) \AA$ at $58.5 \mathrm{GPa}$ (see Table 1). The magnesium atoms are eight-fold coordinated by nitrogen atoms (see Fig. 1), which are arranged in exotic planar infinite zigzag

Table 1 Crystallographic data for the $\mathrm{MgN}_{4}, \beta-\mathrm{Mg}_{2} \mathrm{~N}_{4}$ and $\alpha-\mathrm{Mg}_{2} \mathrm{~N}_{4}$ compounds

\begin{tabular}{|c|c|c|c|}
\hline & $\mathrm{MgN}_{4}$ & $\beta-\mathrm{Mg}_{2} \mathbf{N}_{4}$ & $\alpha-\mathbf{M g}_{2} \mathbf{N}_{4}$ \\
\hline Pressure (GPa) & 58.5 & 58.5 & 0.0001 (1 bar) \\
\hline Space group & Ibam & $P 2 \sqrt{ } n$ & $P 2 \sqrt{ } n$ \\
\hline$a(\AA)$ & $3.5860(13)$ & $7.113(5)$ & 7.5182(9) \\
\hline$b(\AA)$ & $7.526(3)$ & $5.828(6)$ & $6.5426(11)$ \\
\hline$c(\AA)$ & $5.1098(17)$ & $8.800(9)$ & $13.4431(19)$ \\
\hline$\beta\left(^{\circ}\right)$ & 90 & $104.00(7)$ & $130.080(17)$ \\
\hline$V\left(\AA^{3}\right)$ & $137.90(9)$ & $354.0(6)$ & $505.95(18)$ \\
\hline \multirow[t]{12}{*}{ Fractional atomic coordinates $(x ; y ; z)$} & Mg: $(0 ; 0 ; 0.25)$ & Mg1: (0.49079; 0.7820;0.36972) & Mg1: (-0.03444; $-0.26972 ;-0.14194)$ \\
\hline & N1: $(0.6584 ; 0.83344 ; 0.5)$ & Mg2: (0.87494; 1.0122;0.65121) & Mg2: (0.19484; -0.24500; -0.43089) \\
\hline & N2: $(0.2928 ; 0.3059 ; 0.5)$ & Mg3: (0.27163; -0.0478; 0.08387) & Mg3: $(-0.15418 ;-0.47368 ;-0.39088)$ \\
\hline & & Mg4: $(0.73162 ; 1.0462 ; 0.26126)$ & Mg4: (-0.05204; $0.03340 ;-0.31550)$ \\
\hline & & N1: $(0.9146 ; 0.7621 ; 0.4030)$ & N1: (0.2053; 0.2398; -0.29135) \\
\hline & & N2: $(0.5752 ; 0.2424 ; 0.4128)$ & N2: $(0.0799 ;-0.2324 ;-0.32480)$ \\
\hline & & N3: (0.5700; 0.4601; 0.3759) & N3: (0.1944; -0.0551; -0.10797) \\
\hline & & N4: (1.0672; 0.6905; 0.5081) & N4: $(-0.3561 ;-0.1649 ;-0.32605)$ \\
\hline & & N5: (1.0586; 0.7188; 0.6557) & N5: $(-0.0018 ;-0.5637 ;-0.18680)$ \\
\hline & & N6: (0.2820; 0.2132; 0.2432) & N6: (0.1442; 0.3256; -0.39668) \\
\hline & & N7: (0.4332; 0.1114; 0.3403$)$ & N7: $(-0.3255 ; 0.2339 ;-0.45463)$ \\
\hline & & N8: $(0.7714 ; 0.8544 ; 0.4546)$ & N8: $(-0.4397 ;-0.2489 ;-0.43838)$ \\
\hline
\end{tabular}



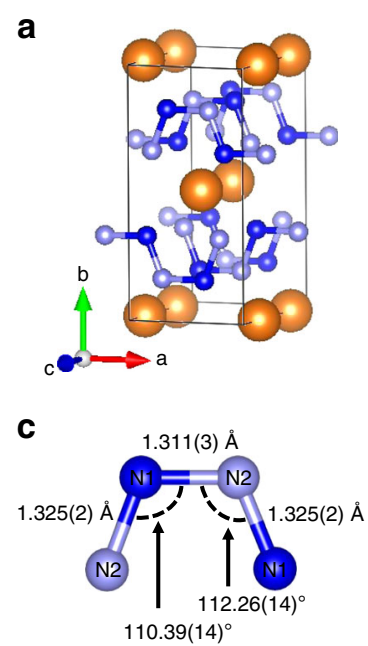

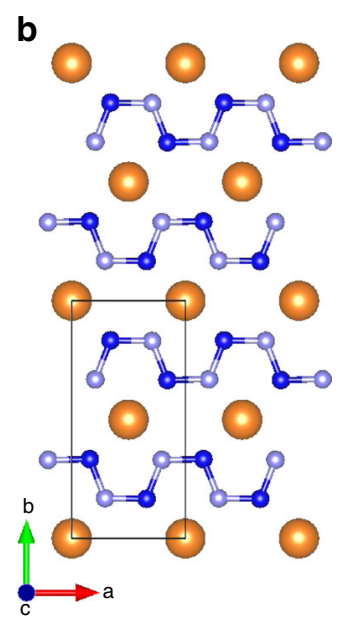

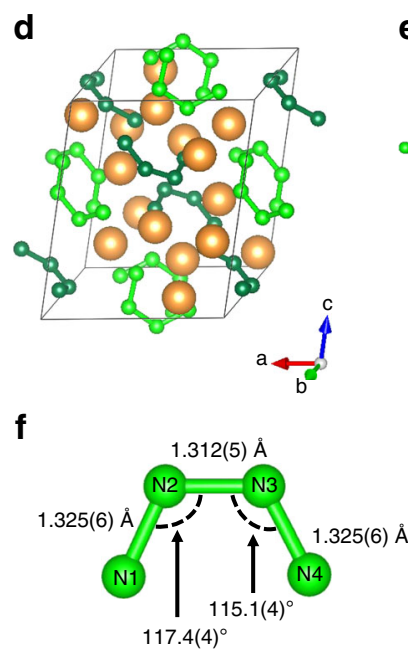
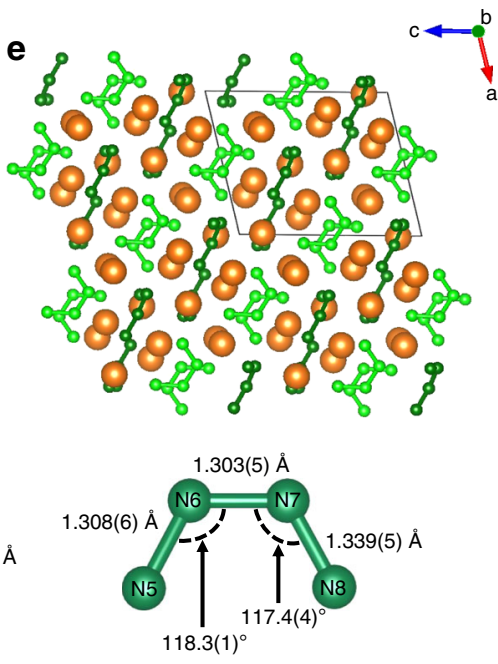

Fig. 1 The crystal structure of the $\mathrm{MgN}_{4}$ and $\beta-\mathrm{Mg}_{2} \mathrm{~N}_{4}$ salts at $58.5 \mathrm{GPa}$. a The unit cell of $\mathrm{MgN}_{4}$ (the light blue, dark blue and orange spheres represent the $\mathrm{N} 1, \mathrm{~N} 2$ and $\mathrm{Mg}$ atoms, respectively); $\mathbf{b}$ a projection of the $\mathrm{MgN}_{4}$ structure along the c-axis, emphasising $1 \mathrm{D}$ chains of nitrogen atoms aligned along the $a$-axis; c a repeating $\mathrm{N}_{4}{ }^{2-}$ subunit of a chain with the $\mathrm{N}-\mathrm{N}$ distances and angles indicated; $\mathbf{d}$ the unit cell of $\beta$ - $\mathrm{Mg}_{2} \mathrm{~N}_{4}$ (the light green and dark green spheres represent the four distinct nitrogen atoms forming the $a-\mathrm{N}_{4}{ }^{4-}$ and $b-\mathrm{N}_{4}{ }^{4-}$ units, respectively, the orange spheres represent $\mathrm{Mg}$ atoms); e a projection of the $\beta-\mathrm{Mg}_{2} \mathrm{~N}_{4}$ structure along the $b$-axis allowing to see the alternating layers of isolated $a-\mathrm{N}_{4}{ }^{4-}$ and $b-\mathrm{N}_{4}{ }^{4-}$ units, intercalated with $\mathrm{Mg}{ }^{2+}$ ions. f The $a-\mathrm{N}_{4}{ }^{4-}$ (left) and $b-\mathrm{N}_{4}{ }^{4-}$ (right) entities with bond lengths and angles indicated

$\mathrm{N}-\mathrm{N}$ chains parallel to the $a$-axis, akin to those found in $\mathrm{FeN}_{4}$ and $\mathrm{ReN}_{8} \cdot x \mathrm{~N}_{2}$ near $110 \mathrm{GPa}^{13,14}$. While the $\mathrm{MgN}_{4}$ compound's crystal chemistry matches the theoretical calculations ${ }^{17-19}$, the measured lattice parameters do not, as the $c$ parameter is double the predicted value and the structure adopts an I-type unit cell, opposite to the calculated $C$-type (see Supplementary Discussion). As shown in Supplementary Fig. 18, by drawing the reciprocal lattice corresponding to the predicted parameter $c(c=2.5549(17)$ $\AA$ ), reflections appear at the mid-point of the lattice vector $c^{*}$; weak but distinctly visible. This means that the $c$ value is actually two times larger $(c=5.1098(17) \AA)$ than the predicted one. The $C$-centering is contradicted by more than 130 experimentally observed reflections. Our DFT calculations reveal the Ibam $\mathrm{MgN}_{4}$ structure to have an enthalpy of about $10 \mathrm{~kJ} / \mathrm{mol}$ lower than the predicted $\mathrm{Cmmm} \mathrm{MgN}_{4}$ solid and to be dynamically stable at 50 GPa (see Supplementary Fig. 10). The full experimental crystallographic data and Raman spectra of $\mathrm{Ibam} \mathrm{MgN}_{4}$ are presented in detail in the Supplementary Table 3 and the Supplementary Fig. 5, along with their further comparison to the theoretically computed data (see Supplementary Fig. 8) ${ }^{17,18}$.

The structure of the high pressure $\mathrm{Mg}_{2} \mathrm{~N}_{4}$ phase (Fig. 1) has a monoclinic $P 2_{1} / n$ symmetry with lattice parameters $a=7.114(3)$ $\AA, b=5.824(2) \AA, c=8.804(4) \AA$ and $\beta=104.04(3)^{\circ}$ at $58.5 \mathrm{GPa}$ (see Table 1). We name it $\beta-\mathrm{Mg}_{2} \mathrm{~N}_{4}$, to distinguish from its ambient pressure modification $\alpha-\mathrm{Mg}_{2} \mathrm{~N}_{4}$ (see below). The unit cell of $\beta-\mathrm{Mg}_{2} \mathrm{~N}_{4}$ contains twelve symmetrically independent atoms, four $\mathrm{Mg}$ and eight $\mathrm{N}$. The atomic arrangement gives rise to an exotic poly- $\mathrm{N}$ entity: a $\mathrm{N}_{4}$ unit with a formal charge of $4-$. The tetranitrogen anion has a surprising cis-like shape, analogous to the nitrogen skeleton in cis-tetrazene-here observed as an isolated molecule 24,25 . The eight nitrogen atoms form two discernable $\mathrm{N}_{4}{ }^{4-}$ units, hereafter named $a-\mathrm{N}_{4}{ }^{4-}$ and $b-\mathrm{N}_{4}{ }^{4-}$, with slight variations in their $\mathrm{N}-\mathrm{N}$ distances. At $58.5 \mathrm{GPa}$, $a-\mathrm{N}_{4}{ }^{4-}$ has bond lengths of 1.325(6), 1.312(5) and 1.325(6) $\AA$ while $b-\mathrm{N}_{4}{ }^{4-}$ has $1.308(6), 1.303(5)$, and 1.339(5) $\AA$, for the first edge bond, the center bond, and the second edge bond, respectively. These modest differences underline a subtle but complex dissimilarity in their chemical environment, namely their proximity and coordination with the $\mathrm{Mg}^{2+}$ ions.
Theoretical insight on the $\boldsymbol{\beta}-\mathbf{M g}_{2} \mathbf{N}_{4}$ salt. The DFT model calculations confirm the $\beta-\mathrm{Mg}_{2} \mathrm{~N}_{4}$ structural model in great detail and hence can confidently be employed to analyze its electronic structure (see Supplementary Table 5 for the complete analysis). The inspection of the total and partial density of states of $\beta$ $\mathrm{Mg}_{2} \mathrm{~N}_{4}$ unambiguously exposes that there is only a very weak covalent bonding between $\mathrm{N}_{4}{ }^{4-}$ entities, and electron difference maps exhibits no charge accumulation between the $\mathrm{Mg}$ and $\mathrm{N}$ atoms. Instead, a strong ionic interaction between the $\mathrm{Mg}$ and $\mathrm{N}$ ions is observed: a Mulliken analysis shows that $\mathrm{Mg}$ is essentially in its charged formal state and a semi-quantitative comparison to $\mathrm{MgCl}_{2}$ implies that the bonding between $\mathrm{Mg}^{2+}$ and the $\mathrm{N}_{4}{ }^{4-}$ entities is even slightly more ionic than between $\mathrm{Mg}^{2+}$ and $\mathrm{Cl}^{-}$. Within the $\mathrm{N}_{4}^{4-}$ entities, the edge and center bonds have the same Mulliken bond population, consistent with their similar bond lengths $(\sim 1.318 \pm 0.015 \AA)$. This theoretical insight exhibits the stark differences in electronic density configuration between the $\mathrm{N}_{4}{ }^{4-}$ entities in $\beta-\mathrm{Mg}_{2} \mathrm{~N}_{4}$ and those previously observed in trans-tetrazene or tetrazadiene complexes, in which the $\mathrm{N}_{4}{ }^{4-}$ units are instead stabilized through strong covalent bonds and bond length disparities denote the single and double bond character of the edge and center bonds, respectively ${ }^{24,25}$.

As the $P 2_{1} / n \beta-\mathrm{Mg}_{2} \mathrm{~N}_{4}$ compound-and its crystal chemistryhad not been predicted by previous theoretical calculations, its enthalpy at $50 \mathrm{GPa}$ was compared to the enthalpies of the $\mathrm{P6}_{3} / \mathrm{mcm}$ and the $\mathrm{Cmcm} \mathrm{MgN}_{2}$ structures that had been predicted $^{17,18}$. We found that $P 2_{1} / n \beta-\mathrm{Mg}_{2} \mathrm{~N}_{4}$ is energetically competitive with the two predicted structure as all were found to be equal within $5 \mathrm{~kJ} / \mathrm{mol}$, which is less than the uncertainty of our calculations. The Raman spectrum of $P 2_{1} / n \beta-\mathrm{Mg}_{2} \mathrm{~N}_{4}$ was also computed and reproduces well the experimental spectrum recorded at $49.7 \mathrm{GPa}$, as seen in Supplementary Fig. 11.

Recovery of the $\alpha-\mathrm{Mg}_{2} \mathrm{~N}_{4}$ salt to ambient conditions. After the synthesis of the $\beta-\mathrm{Mg}_{2} \mathrm{~N}_{4}$ compound, the DACs were slowly decompressed and Raman as well as XRD measurements were performed at each step of the decompression to track possible changes. Both the vibrational modes (Fig. 2) and the lattice 

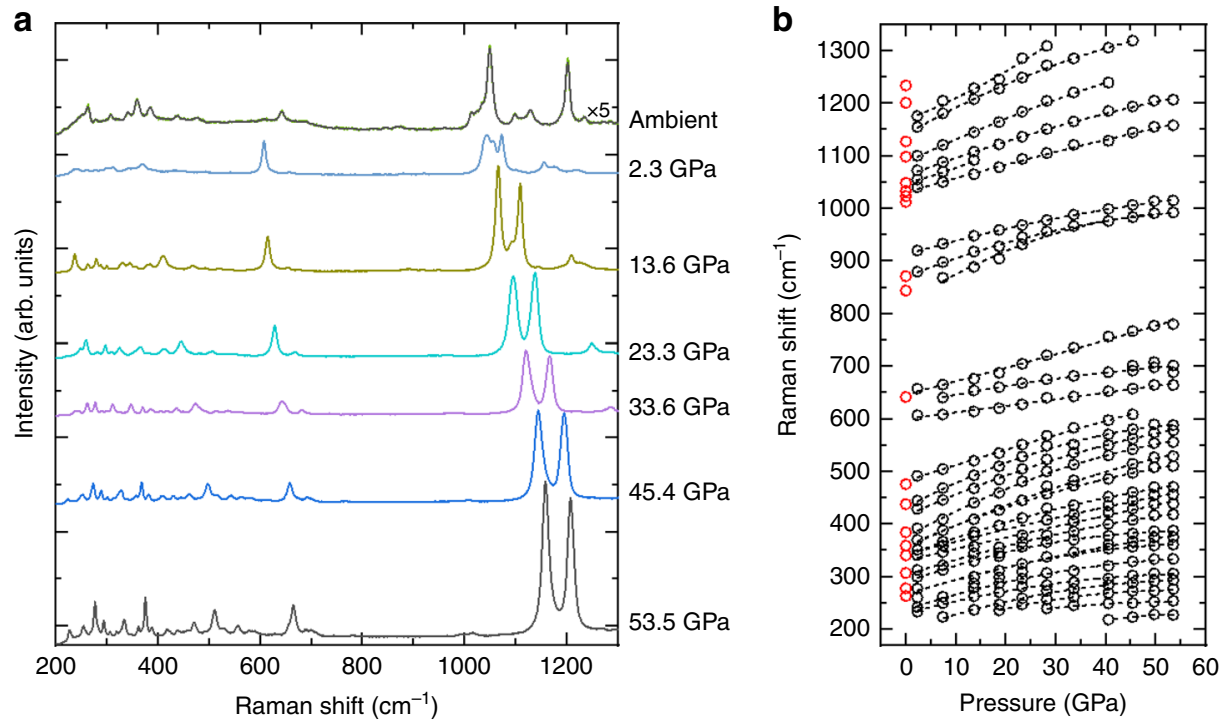

Fig. 2 Evolution of the Raman modes of the $\mathrm{Mg}_{2} \mathrm{~N}_{4}$ compounds with pressure. a Typical Raman spectra of $\mathrm{Mg}_{2} \mathrm{~N}_{4}$ taken during decompression from 53.5 GPa to ambient conditions. The spectra at ambient conditions is markedly different than those at higher pressures, evidencing a phase transition. b Pressure dependence of the Raman modes' frequencies, which evolve smoothly and continuously down to $2.3 \mathrm{GPa}$. Red dots indicate the modes at ambient pressure. The spectra are offset along the $y$-axis for clarity and $\times 5$ indicates that the spectrum is five-times magnified

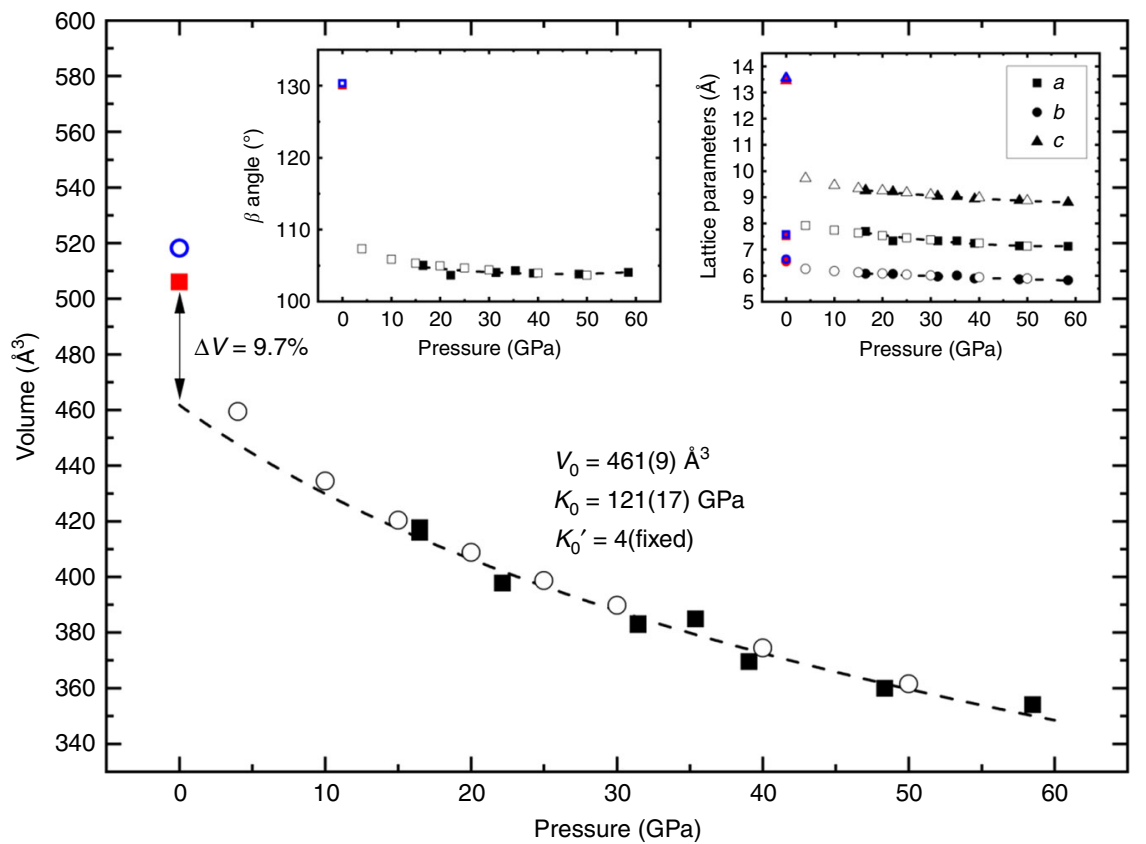

Fig. 3 Unit cell volume of the $\mathrm{Mg}_{2} \mathrm{~N}_{4}$ compounds as a function of pressure. All solid and open symbols are experimental and theoretical data points, respectively, while the dashed line is the fit of the experimental PV data of the $\beta-\mathrm{Mg}_{2} \mathrm{~N}_{4}$ compound using the second order Birch-Murnaghan equation of state (BM2 EoS) $\left(V_{0}=461(9) \AA^{3}\right.$ and $\left.K_{0}=121(17) \mathrm{GPa}\right)$. Red and blue symbols are the experimental and theoretical, respectively, unit cell volume of $\alpha-\mathrm{Mg}_{2} \mathrm{~N}_{4}$ at ambient pressure. The extrapolation of the experimental equation of state suggests a volume jump of $9.7 \%$ between the high pressure $\beta-\mathrm{Mg}_{2} \mathrm{~N}_{4}$ and the ambient pressure $\alpha-\mathrm{Mg}_{2} \mathrm{~N}_{4}$ phases. The fit of the theoretical PV data using the BM2 EoS gives for $\beta-\mathrm{Mg}_{2} \mathrm{~N}_{4} V_{0}=470.23 \AA^{3}$ and $K_{0}=$ $110.83 \mathrm{GPa}$. The theoretical volume difference is thus of $10.2 \%$ between the calculated $\beta-\mathrm{Mg}_{2} \mathrm{~N}_{4}$ and $\alpha-\mathrm{Mg}_{2} \mathrm{~N}_{4}$. The insets show the dependence of the unit cell parameters on pressure. The full (open) black and full red (open blue) symbols represent experimental (theoretical) data from the $\beta$ and $\alpha$ phases of $\mathrm{Mg}_{2} \mathrm{~N}_{4}$. The slightly higher volume obtained from the DFT calculations, compared to the experimental values, shows the underbinding in GGA26

parameters (Fig. 3 and Supplementary Tables 5-8) vary smoothly with pressure, displaying no sign of a chemical reaction, decomposition or phase transition down to $2.3 \mathrm{GPa}$. However, the opening of the DACs in air leads to the loss of the Raman signal from the sample, caused by its deterioration, presumably following a chemical reaction with the water or the oxygen in air (see Supplementary Fig. 13). Therefore, at various pressures below $18 \mathrm{GPa}$, the DACs were opened in a glovebox under an inert atmosphere of argon. After the release of pressure, the DACs were closed, still in the glovebox, so that the samples were preserved in an inert atmosphere at ambient pressure. Microphotographs of a sample before and after the complete release of pressure are shown in Supplementary Fig. 12. The Raman spectra of these decompressed samples appeared to be different from 


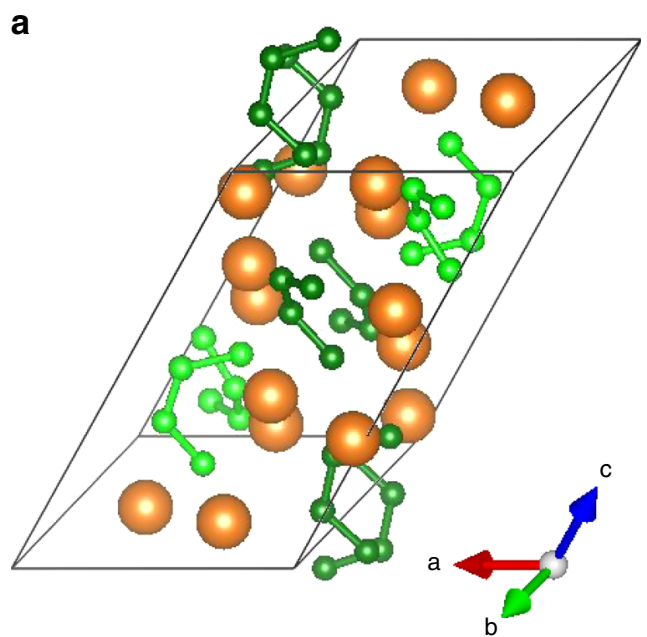

b
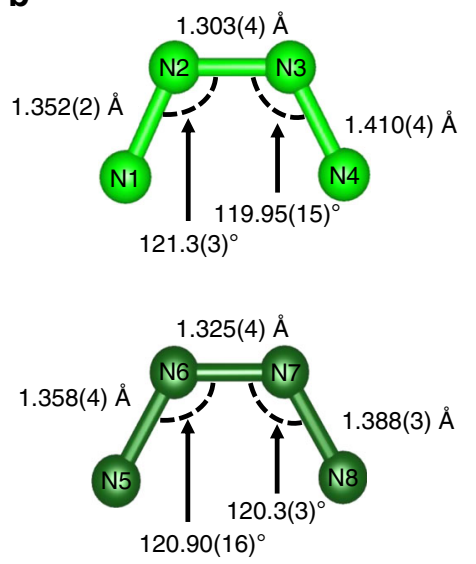

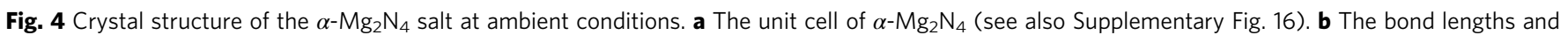

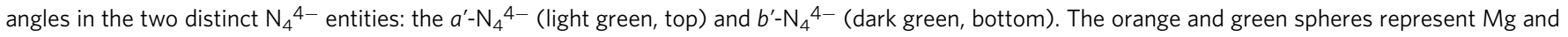
$\mathrm{N}$ atoms, respectively

those previously observed for $\beta-\mathrm{Mg}_{2} \mathrm{~N}_{4}$. To clarify their structure and chemical composition, the samples were investigated employing single-crystal and powder XRD.

The single-crystal XRD analysis revealed a polymorph of $\mathrm{Mg}_{2} \mathrm{~N}_{4}\left(\alpha-\mathrm{Mg}_{2} \mathrm{~N}_{4}\right)$, which still contains two distinct $\mathrm{N}_{4}{ }^{4-}$ units (Fig. 4). Its structural details are found in Table 1 while the full crystallographic data are provided in the Supplementary Table 9. A Le Bail refinement performed on a diffractogram collected from a $\alpha-\mathrm{Mg}_{2} \mathrm{~N}_{4}$ sample at ambient conditions (i.e., after the release of gaseous $\mathrm{N}_{2}$ ) revealed no other phases, as seen in Supplementary Fig. 15. The structure has the same monoclinic symmetry $\left(P 2_{1} / n\right.$ space group) with lattice parameters of $a=7.5182(9) \AA, b=$ 6.5426(11) $\AA, c=13.4431(19) \AA$, and $\beta=130.080(17)^{\circ}$. The unit cell is strongly deformed in comparison to the high pressure polymorph, with a large increase of the unit cell parameters $c$ and $\beta$ as well as a decrease of the unit cell parameter $a$. The stacking of the $\mathrm{N}_{4}{ }^{4-}$ units in $\alpha-\mathrm{Mg}_{2} \mathrm{~N}_{4}$ also differs from that in the high pressure $\beta-\mathrm{Mg}_{2} \mathrm{~N}_{4}$ phase, as shown in Supplementary Fig. 17. The structural model is again confirmed in great detail by DFT calculations, which also show that the $\alpha-\mathrm{Mg}_{2} \mathrm{~N}_{4}$ is more stable than the $\beta-\mathrm{Mg}_{2} \mathrm{~N}_{4}$ by $10 \mathrm{~kJ} / \mathrm{mol}$ at ambient conditions (see Supplementary Discussion).

In the $\alpha-\mathrm{Mg}_{2} \mathrm{~N}_{4}$ phase, both of the $\mathrm{N}_{4}{ }^{4-}$ entities display slightly longer $\mathrm{N}-\mathrm{N}$ distances. In $a^{\prime}-\mathrm{N}_{4}{ }^{4-}$, the bond lengths are of 1.352(2), $1.303(4)$ and $1.410(4) \AA$, while in $b^{\prime}-\mathrm{N}_{4}{ }^{4-}$ they are $1.358(4), 1.325$ (4) and 1.388(3) $\AA$, for the first edge bond, the center bond and the second edge bond, respectively. This resembles the bonds lengths measured in some tetrazadiene complexes (namely [ $\operatorname{Ir}(\mathrm{RNNNNR})$ (CO) $\left.\left(\mathrm{PPh}_{3}\right)_{2}\right]\left[\mathrm{BF}_{4}\right]$, where $\mathrm{R}$ is $\left.4-\mathrm{FC}_{6} \mathrm{H}_{4}\right)^{25}$. The DFT calculations show that there are essentially no differences in the character of chemical bonding between the $\mathrm{Mg}^{2+}$ and the $\mathrm{N}_{4}{ }^{4-}$ entities in $\alpha-\mathrm{Mg}_{2} \mathrm{~N}_{4}$ and $\beta-\mathrm{Mg}_{2} \mathrm{~N}_{4}$, implying that it is still strongly ionic. The energy released upon the decomposition of $\alpha-\mathrm{Mg}_{2} \mathrm{~N}_{4}$ into $\mathrm{Mg}_{3} \mathrm{~N}_{2}$ and molecular $\mathrm{N}_{2}$ is calculated to be about $1.9 \mathrm{~kJ} / \mathrm{g}$, which is about two times less than known for $\mathrm{TNT}^{27,28}$.

The $\alpha-\mathrm{Mg}_{2} \mathrm{~N}_{4}$ salt encapsulated in inert atmosphere persisted at ambient conditions for several months, as evidenced by singlecrystal X-ray diffraction and Raman spectroscopy (see Supplementary Fig. 14). Although Raman spectroscopy measurements suggest the stability of the $\mathrm{MgN}_{4}$ compound at least down to 0.9 GPa (see Supplementary Fig. 9), it was not detected by XRD at a pressure other than $58.5 \mathrm{GPa}$. The DFT calculations suggest this compound to be elastically unstable at ambient conditions, as the $c_{44}$ and $c_{66}$ parameters were calculated to have values below $0 \mathrm{GPa}$. Further experiments and calculations are underway to conclusively determine the stability domain of $\mathrm{MgN}_{4}$.

The high pressure investigation of the $\mathrm{Mg}-\mathrm{N}$ system unveiled an exotic and unexpected chemistry. First, a compound featuring infinite $1 \mathrm{D}$ polynitrogen chains was obtained near $50 \mathrm{GPa}$-half of the pressure previously thought to be required ${ }^{13,14}$. Second, in the same pressure domain, previously unknown $\left(\mathrm{N}_{4}\right)^{4-}$ polynitrogen anions were synthesized and stabilized in the simple $\mathrm{Mg}_{2} \mathrm{~N}_{4}$ salt. Its high pressure polymorph $\left(\beta-\mathrm{Mg}_{2} \mathrm{~N}_{4}\right)$ undergoes a phase transition on pressure release, but the $\left(\mathrm{N}_{4}\right)^{4-}$ polynitrogen units persist in its low pressure polymorph $\left(\alpha-\mathrm{Mg}_{2} \mathrm{~N}_{4}\right)$, adding a unique $\left(\mathrm{N}_{4}\right)^{4-}$ anion to a yet very short list of poly- $\mathrm{N}$ entities bulk stabilized at ambient conditions. The tetranitrogen anion can spark further research for producing improved energetic polynitrogen compounds. Moreover, these results underline the potential and possibilities that are enabled by high pressure chemistry, along with its applicability to produce compounds compatible with ambient conditions.

\section{Methods}

Sample preparation. A few pure magnesium flakes of typically about $10 \times 10 \times$ $5 \mu \mathrm{m}^{3}$ in size were positioned on one of the diamond anvils, with culet diameter of $250 \mu \mathrm{m}$. Rhenium was used as the gasket material. A small ruby chip was loaded along with the sample and used to determine the pressure inside the pressure chamber ${ }^{29}$. The diamond anvil cell (DAC) pressure chamber was then loaded with pure molecular nitrogen ( 1200 bars) in a high pressure vessel. Pure nitrogen, acting as both a pressure transmitting medium and a reagent, was always largely in excess with respect to magnesium.

Throughout the procedure, care was taken to minimize the exposure of $\mathrm{Mg}$ to air. On average, the magnesium flakes were exposed for about 20 min to air during the complete loading procedure. According to previous investigations ${ }^{30}$, an exposure of this time span is expected to produce a $20-50 \mathrm{~nm}$ protective oxide film at the surface of $\mathrm{Mg}$, which prevents further chemical reaction between $\mathrm{Mg}$ and elements in the air. Considering the volume of the loaded $\mathrm{Mg}$ pieces (about $10 \times$ $10 \times 5 \mu \mathrm{m}^{3}$ ), the very limited amounts of $\mathrm{Mg}$ oxides are not thought to play a role in the observed chemical reactions between nitrogen and magnesium. Indeed, sample characterization by Raman spectroscopy and X-ray diffraction never revealed vibrational modes or diffraction peaks that could be attributed to a solid other than a $\mathrm{Mg}-\mathrm{N}$ compound.

Raman spectroscopy. Sample characterization was achieved in part by confocal Raman spectroscopy measurements performed with a LabRam spectrometer equipped with a $\times 50$ Olympus objective. Sample excitation was accomplished using a continuous $\mathrm{He}-\mathrm{Ne}$ laser $(632.8 \mathrm{~nm}$ line) with a focused laser spot of about $2 \mu \mathrm{m}$ in diameter. The 
Stokes Raman signal was collected in a backscattering geometry by a CCD coupled to an $1800 \mathrm{l} / \mathrm{mm}$ grating, allowing a spectral resolution of approximately $2 \mathrm{~cm}^{-1}$. At ambient pressure, after the release of gaseous molecular nitrogen, the full power Raman laser-4.6 $\mathrm{mW}$ incident on the DAC-resulted in the decomposition of the $\alpha-\mathrm{Mg}_{2} \mathrm{~N}_{4}$ crystallites; the irradiated spots becoming dark and no longer displaying the $\alpha-\mathrm{Mg}_{2} \mathrm{~N}_{4}$ vibrational modes. To avoid this, the laser power was reduced by employing neutral filters by a factor of approximately $6(0.75 \mathrm{~mW}$ on the DAC).

X-ray diffraction. The X-ray diffraction studies were performed at the P02.2 beamline $(\lambda=0.2901 \AA)$ at PETRA III. In order to determine the sample position on which the single crystal X-ray diffraction acquisition is obtained, a full X-ray diffraction mapping of the experimental cavity is performed. The sample position displaying the most single crystal reflections belonging to the phase of interest is chosen for the collection, in step-scans of $0.5^{\circ}$ from $-36^{\circ}$ to $+36^{\circ} \omega$, of the single crystal X-ray diffraction data. The CrysAlis ${ }^{P r o}$ software $^{31}$ is utilized for the single crystal data analysis. The analysis procedure includes the peak search, the removal of the diamond anvils' parasitic reflections, finding reflections belonging to a unique single crystal, the unit cell determination and the data integration. The crystal structures are then solved and refined within the JANA2006 software ${ }^{32}$. The procedure for single crystal X-ray diffraction data acquisition and analysis was previously demonstrated and successfully employed ${ }^{13,14,33,34}$. Powder X-ray diffraction was also performed to verify the chemical homogeneity of the samples. The powder X-ray data was integrated with Dioptas ${ }^{35}$ and analyzed with the XRDA software $^{36}$. Le Bail refinements employing a powder X-ray diffraction pattern was accomplished with the FullProf software ${ }^{37}$.

Laser-heating. The double-sided sample laser-heating was performed at our home laboratory at the Bayreuth Geoinstitut using two YAG lasers. Pure magnesium, which is metallic, was employed as the YAG laser absorber. Temperatures were accurately determined from the sample's blackbody radiation ${ }^{38}$. Samples were heated between 10 and $15 \mathrm{~min}$ and moved under the YAG beams to evenly heat the whole magnesium piece.

Atomistic modeling. Density functional theory (DFT) calculations have been performed using the CASTEP code ${ }^{39}$. The code is an implementation of KohnSham DFT based on a plane wave basis set in conjunction with pseudopotentials. The plane-wave basis set is unbiased (as it is not atom-centered) and does not suffer from the problem of basis-set superposition error unlike atom-centered basis sets. It also makes converged results straightforward to obtain in practice, as the basis set convergence is controlled by a single adjustable parameter, the plane wave cut-off. Pseudopotentials were either norm-conserving or ultrasoft, and were generated using the PBE exchange-correlation functional ${ }^{40}$ using the 'on the fly' parameters included in the CASTEP 2019 distribution. These pseudopotentials have been shown to be very accurate and are very well suited for the calculations carried out here ${ }^{41}$. The Brillouin-zone integrals were performed using MonkhorstPack grids ${ }^{42}$ with spacings between grid points of less than $0.02 \AA^{-1}$. Full geometry optimizations of the unit cell parameters and the internal coordinates were performed until forces were converged to $<0.01 \mathrm{eV} / \AA$ and the residual stress was $<0.02$ GPa. Phonon dispersion curves and Raman spectra were computed using linear response theory ${ }^{43}$ as implemented in CASTEP ${ }^{44}$. The population analysis was carried out as implemented in CASTEP ${ }^{45}$. Band gaps were obtained from band structure calculations. DFT-GGA calculations tend to systematically underestimate the band gap.

We benchmarked our model calculations by comparison of our results to the experimentally determined ambient conditions structure of $\mathrm{Mg}_{3} \mathrm{~N}_{2}$, which adopts the space group Ia31 and has a lattice parameter of $9.9528(1) \AA^{46}$. Our calculations gave $a=10.0127 \AA$, thus showing the often observed slight underbinding in GGA calculations. The experimental Raman spectrum ${ }^{47}$ is well reproduced, as shown in Supplementary Fig. 3.

\section{Data availability}

The details of the crystal structure investigations may be obtained from the Cambridge Crystallographic Data Centre (CCDC), 12 Union Road, CB2 1EZ Cambridge, United Kingdom (fax: + 44 (0)1223 336033; e-mail: admin@ccdc.cam.ac.uk) on quoting the deposition numbers CSD 1918150-1918155 and CSD 19182019. The data that support the findings of this study are available from the corresponding author upon reasonable request.

Received: 14 June 2019; Accepted: 16 September 2019; Published online: 04 October 2019

\section{References}

1. Klapötke, T. M. New Nitrogen-Rich High Explosives. in High Energy Density Materials 85-121 (Springer, 2007).
2. Christe, K. O. Polynitrogen chemistry enters the ring. Science 355, 351-351 (2017).

3. Vij, A., Pavlovich, J. G., Wilson, W. W., Vij, V. \& Christe, K. O. Experimental detection of the pentaazacyclopentadienide (pentazolate) anion, cyclo-N5Angew. Chem. - Int. Ed. 41, 3051-3054 (2002).

4. Christe, K. O., Wilson, W. W., Sheehy, J. A. \& Boatz, J. A. N5+: a novel homoleptic polynitrogen ion as a high energy density material. Angew. Chem. Int. Ed. 38, 2004-2009 (1999).

5. $\mathrm{Xu}, \mathrm{Y}$. et al. A series of energetic metal pentazolate hydrates. Nature $\mathbf{5 4 9}$, 78-81 (2017)

6. Jiang, C. et al. Synthesis and characterization of the pentazolate anion cycloN5- in (N5)6(H3O)3(NH4)4Cl. Science 355, 347-376 (2017).

7. Zhang, W. et al. Stabilization of the Pentazolate Anion in a Zeolitic Architecture with Na20N60 and Na24N60Nanocages. Angew. Chem. - Int. Ed. 57, 2592-2595 (2018)

8. Zhang, C. et al. A symmetric $\mathrm{Co}(\mathrm{N} 5) 2(\mathrm{H} 2 \mathrm{O}) 44 \mathrm{H} 2 \mathrm{O}$ high-nitrogen compound formed by cobalt(II) cation trapping of a cyclo-N5- anion. Angew. Chem. - Int. Ed. 56, 4512-4514 (2017).

9. Cacace, F., de Petris, G. \& Troiani, A. Experimental detection of tetranitrogen. Science 295, 480-481 (2002).

10. Knight, L. B. et al. ESR and ab initio theoretical studies of the cation radicals $14 \mathrm{~N} 4+$ and $15 \mathrm{~N} 4+$ : The trapping of ion-neutral reaction products in neon matrices at 4 K. J. Chem. Phys. 87, 885-897 (1987).

11. Thompson, W. E. \& Jacox, M. E. The vibrational spectra of molecular ions isolated in solid neon. II. O4+ and O4-. J. Chem. Phys. 91, 3826-3837 (1989).

12. Eremets, M. I., Gavriliuk, A. G., Trojan, I. A., Dzivenko, D. A. \& Boehler, R. Single-bonded cubic form of nitrogen. Nat. Mater. 3, 558-563 (2004).

13. Bykov, M. et al. Fe-N system at high pressure reveals a compound featuring polymeric nitrogen chains. Nat. Commun. 9, 2756 (2018).

14. Bykov, M. et al. High-pressure synthesis of a nitrogen-rich inclusion compound ReN $8 \cdot$ x N 2 with conjugated polymeric nitrogen chains. Angew. Chem. Int. Ed. 57, 9048-9053 (2018).

15. Steele, B. A. et al. High-pressure synthesis of a pentazolate salt. Chem. Mater. 29, 735-741 (2017).

16. Laniel, D., Weck, G., Gaiffe, G., Garbarino, G. \& Loubeyre, P. High-pressure synthesized lithium pentazolate compound metastable under ambient conditions. J. Phys. Chem. Lett. 9, 1600-1604 (2018).

17. Yu, S. et al. Emergence of novel polynitrogen molecule-like species, covalent chains, and layers in magnesium-nitrogen $\mathrm{Mg} \times \mathrm{N}$ y phases under high pressure. J. Phys. Chem. C. 121, 11037-11046 (2017).

18. Wei, S. et al. Alkaline-earth metal $(\mathrm{Mg})$ polynitrides at high pressure as possible high-energy materials. Phys. Chem. Chem. Phys. 19, 9246-9252 (2017).

19. Xia, K. et al. Pressure-stabilized high-energy-density alkaline-earth-metal pentazolate salts. J. Phys. Chem. C 123, 10205-10211 (2019).

20. Hao, J. et al. Structural phase transformations of $\mathrm{Mg} 3 \mathrm{~N} 2$ at high pressure: experimental and theoretical studies. Inorg. Chem. 48, 9737-9741 (2009).

21. Stinton, G. W. et al. Equation of state and high-pressure/high-temperature phase diagram of magnesium. Phys. Rev. B 90, 134105 (2014).

22. Eremets, M. I. et al. Structural transformation of molecular nitrogen to a single-bonded atomic state at high pressures. J. Chem. Phys. 121, 11296-11300 (2004).

23. Olijnyk, H. High pressure x-ray diffraction studies on solid N2 up to $43.9 \mathrm{GPa}$. J. Chem. Phys. 93, 8968 (1990).

24. Holleman, A. F. \& Wiberg, E. Lehrbuch der Anorganischen Chemie. (Walter de Gruyter, 1995).

25. Moore, D. S. \& Robinson, S. D. Catenated nitrogen ligands part I.1 Transition metal derivatives of triazenes, tetrazenes, tetrazadienes, and pentazadienes. Adv. Inorg. Chem. 30, 1-68 (1986).

26. Winkler, B. \& Milman, V. Density functional theory based calculations for high pressure research. Z. f.ür. Krist. - Cryst. Mater. 229, 112-122 (2014).

27. Lindsay, C. M. \& Fajardo, M. E. The quest for greater chemical energy storage in energetic materials: grounding expectations. AIP Conf. Proc. 1793, 040023 (2017).

28. Zhang, J., Oganov, A. R., Li, X. \& Niu, H. Pressure-stabilized hafnium nitrides and their properties. Phys. Rev. B 95, 020103 (2017).

29. Dewaele, A., Torrent, M., Loubeyre, P. \& Mezouar, M. Compression curves of transition metals in the Mbar range: experiments and projector augmentedwave calculations. Phys. Rev. B 78, 104102 (2008).

30. Nordlien, J. H. Morphology and structure of oxide films formed on magnesium by exposure to air and water. J. Electrochem. Soc. 142, 3320 (1995).

31. Rigaku Oxford Diffraction. CrysAlisPro Software system. (2015)

32. Petrícek, V., Dušek, M. \& Palatinus, L. Crystallographic computing system JANA2006: General features. Z. fur Krist. 229, 345-352 (2014).

33. Bykov, M. et al. Synthesis of FeN4 at $180 \mathrm{GPa}$ and its crystal structure from a submicron-sized grain. Acta Crystallogr. Sect. E Crystallogr. Commun. 74, 1392-1395 (2018). 
34. Bykova, E. Single-crystal X-ray diffraction at extreme conditions in mineral physics and material sciences. (University of Bayreuth, 2015).

35. Prescher, C. \& Prakapenka, V. B. DIOPTAS: a program for reduction of twodimensional X-ray diffraction data and data exploration. High. Press. Res. 35, 223-230 (2015).

36. Desgreniers, S. \& Lagarec, K. XRDA: a program for energy-dispersive X-ray diffraction analysis on a PC. J. Appl. Crystallogr. 27, 432-434 (1994).

37. Rodriguez-Carvajal, J. Recent advances in magnetic structure determination neutron powder diffraction. Phys. B Condens. Matter 192, 55-69 (1993).

38. Mezouar, M. et al. Methodology for in situ synchrotron X-ray studies in the laser-heated diamond anvil cell. High. Press. Res. 37, 170-180 (2017).

39. Clark, S. J. et al. First principles methods using CASTEP. Z. fur Krist. 220, 567-570 (2005).

40. Perdew, J. P., Burke, K. \& Ernzerhof, M. Generalized gradient approximation made simple. Phys. Rev. Lett. 77, 3865-3868 (1996).

41. Lejaeghere, K. et al. Reproducibility in density functional theory calculations of solids. Science 351, 1-7 (2016).

42. Monkhorst, H. J. \& Pack, J. D. Special points for Brillouin-zone integrations. Phys. Rev. B 13, 5188-5192 (1976).

43. Baroni, S., de Gironcoli, S., Dal Corso, A. \& Giannozzi, P. Phonons and related crystal properties from density-functional perturbation theory. Rev. Mod. Phys. 73, 515-562 (2001).

44. Refson, K., Tulip, P. R. \& Clark, S. J. Variational density-functional perturbation theory for dielectrics and lattice dynamics. Phys. Rev. B Condens. Matter Mater. Phys. 73, 1-12 (2006).

45. Segall, M. D., Pickard, C. J., Shah, R. \& Payne, M. C. Population analysis in plane wave electronic structure calculations. Mol. Phys. 89, 571-577 (1996).

46. Partin, D. E., Williams, D. J. \& O'Keeffe, M. The crystal structures of $\mathrm{Mg}_{3} \mathrm{~N}_{2}$ and $\mathrm{Zn}_{3} \mathrm{~N}_{2}$. J. Solid State Chem. 132, 56-59 (1997).

47. Heyns, A. M., Prinsloo, L. C., Range, K. J. \& Stassen, M. The vibrational spectra and decomposition of $\alpha$-calcium nitride $\left(\alpha-\mathrm{Ca}_{3} \mathrm{~N}_{2}\right)$ and magnesium nitride $\left(\mathrm{Mg}_{3} \mathrm{~N}_{2}\right)$. J. Solid State Chem. 137, 33-41 (1998).

\section{Acknowledgements}

The authors acknowledge the Deutsches Elektronen-Synchrotron (DESY, PETRA III) for provision of beamtime at the P02.2 beamline. Alexander Kurnosov is greatly thanked for helping with sample loadings employing the high pressure gas loader. D.L. thanks the Alexander von Humboldt Foundation for financial support. N.D. and L.D. thank the Federal Ministry of Education and Research, Germany (BMBF, grants no. 5K16WC1 and no. 05K19WC1) and the Deutsche Forschungsgemeinschaft (DFG projects DU 954-11/1, DU 393-9/2, and DU 393-13/1) for financial support. B.W. gratefully acknowledges funding by the DFG in the framework of the research unit DFG FOR2125 and within projects WI1232.

\section{Author contributions}

D.L., L.D. and N.D. conceptualized the research, D.L., E.K., T.F., M.B. and E.B. participated to the experimental data collection, B.W. performed all theoretical calculations and carried out their analysis, D.L., E.K, T.F. and M.B. conducted the sample laserheating, D.L. analyzed the experimental results, wrote the original draft and created the figures, D.L., B.W., L.D. and N.D. reviewed and edited the draft.

\section{Competing interests}

The authors declare no competing interests.

\section{Additional information}

Supplementary information is available for this paper at https://doi.org/10.1038/s41467019-12530-w.

Correspondence and requests for materials should be addressed to D.L.

Peer review information Nature Communications thanks Karl Christe, Kamil Dziubek, and the other, anonymous, reviewer for their contribution to the peer review of this work. Peer reviewer reports are available.

Reprints and permission information is available at http://www.nature.com/reprints

Publisher's note Springer Nature remains neutral with regard to jurisdictional claims in published maps and institutional affiliations.

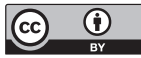

Open Access This article is licensed under a Creative Commons Attribution 4.0 International License, which permits use, sharing, adaptation, distribution and reproduction in any medium or format, as long as you give appropriate credit to the original author(s) and the source, provide a link to the Creative Commons license, and indicate if changes were made. The images or other third party material in this article are included in the article's Creative Commons license, unless indicated otherwise in a credit line to the material. If material is not included in the article's Creative Commons license and your intended use is not permitted by statutory regulation or exceeds the permitted use, you will need to obtain permission directly from the copyright holder. To view a copy of this license, visit http://creativecommons.org/ licenses/by/4.0/.

(C) The Author(s) 2019 